\title{
Relación entre las masas de agua y la composición y distribución de las larvas de peces en el mar de Alborán (Mediterráneo occidental) y estrecho de Gibraltar
}

\author{
Water masses and fish larvae in the Alboran Sea (Western Mediterranean) and Strait of Gibraltar \\ Paulo Mafalda Jr. ${ }^{1}$, Juan Perez de Rubin² y Christiane Sampaio de Souza ${ }^{1}$ \\ ${ }^{1}$ Laboratório de Plâncton. Instituto de Biologia. Universidade Federal da Bahia. 40210-020. Salvador, Bahia, Brasil \\ ${ }^{2}$ Centro Oceanográfico de Málaga. Instituto Español de Oceanografía. Muelle Pesquero s/n. 29640. Fuengirola (Málaga), España \\ pomafa@ufba.br
}

\begin{abstract}
In this paper the relationship between interannual changes in the water masses and fish larvae composition and distribution at the Alboran Sea and Strait of Gibraltar were analysed. Among the dominant taxa only two neritic taxa (Sparidae and Cepola rubescens) and five oceanic taxa (Ceratoscopelus maderensis, Maurolicus muelleri, Benthosema glaciale, Hygophum benoiti and Capros aper) were present in both surveys. At sampling station level, pronounced interannual substitutions were detected in dominant taxa and water masses. The clusters reflected time-space changes in both larval communities (neritic / oceanic), with a differing horizontal zonation: latitudinal partitioning in 1993 and a longitudinal partitioning in 1994. These results indicate that the structure of the larval associations is modulated by changes in the local hydrodynamics.
\end{abstract}

Key words: Fish larvae, Alboran Sea, Strait of Gibraltar, water masses

\section{Introducción}

El estrecho de Gibraltar comunica el océano Atlántico con el mar Mediterráneo (Fig.1). Las aguas oceánicas de la cercana zona noroeste del mar de Alborán se caracterizan por la presencia del 'chorro' de agua atlántica (AA) entrante y el límite norte de un remolino anticiclónico, con un intenso frente asociado al borde septentrional, y la existencia de zonas de divergencia. Para éstas se han propuesto varios mecanismos de afloramiento (Sarhan et al. 2000) y se han encontrado diferencias en la naturaleza del agua aflorada: surgencias de aguas mediterráneas en el talud (Ballester \& Zavatti 1983) y de aguas atlánticas subsuperficiales (Gil 1985).

La masa de agua AA domina la dinámica del área, aportando nutrientes (Gómez et al. 2000) y transportando ictioplancton proveniente del área atlántica. Las
Resumen.- En este estudio se analizan las relaciones entre la composición y distribución de las larvas de peces y los cambios interanuales de las masas de agua en el mar de Alborán y Estrecho de Gibraltar. Entre los taxones dominantes solamente dos taxones costeros (Sparidae y Cepola rubescens) y cinco taxones oceánicos (Ceratoscopelus maderensis, Maurolicus muelleri, Benthosema glaciale, Hygophum benoiti y Capros aper), estuvieron presentes en las dos prospecciones estivales. A nivel de estación de muestreo, los resultados mostraron notorias sustituciones interanuales, tanto en el taxón dominante, como en la masa de agua. Los dendrogramas reflejaron cambios espacio-temporales en las comunidades larvarias neríticas / oceánicas y mostraron una diferente zonación horizontal: partición latitudinal en 1993 y longitudinal en 1994. Estos resultados indican que la estructura de las asociaciones larvarias es regulada por los cambios en la hidrodinámica local.

Palabras clave: Larvas de peces, mar de Alborán, estrecho de Gibraltar, masas de agua variaciones diarias de presión atmosférica en el área influyen en la variabilidad del flujo del AA a través del estrecho (García Lafuente et al. 1998), y los cambios en su velocidad afectan considerablemente la amplitud y localización del giro anticiclónico permanente en la zona occidental del mar de Alborán (Cheney \& Doblar 1982, Parada \& Cantón 1998). La influencia directa del AA sobre la zona noreste de Alborán generalmente solo afecta hasta el talud, pues en la plataforma y en el sector más nerítico las corrientes locales están regidas principalmente por el régimen de vientos (Cano \& García Lafuente 1991).

Las cambiantes particularidades hidrológicas descritas provocan una gran variabilidad temporal de las condiciones ambientales en la zona y suelen ser responsables de altas concentraciones de pigmentos fotosintéticos en verano (Cortés et al. 1985), de una elevada producción biológica local (Rodríguez et al. 


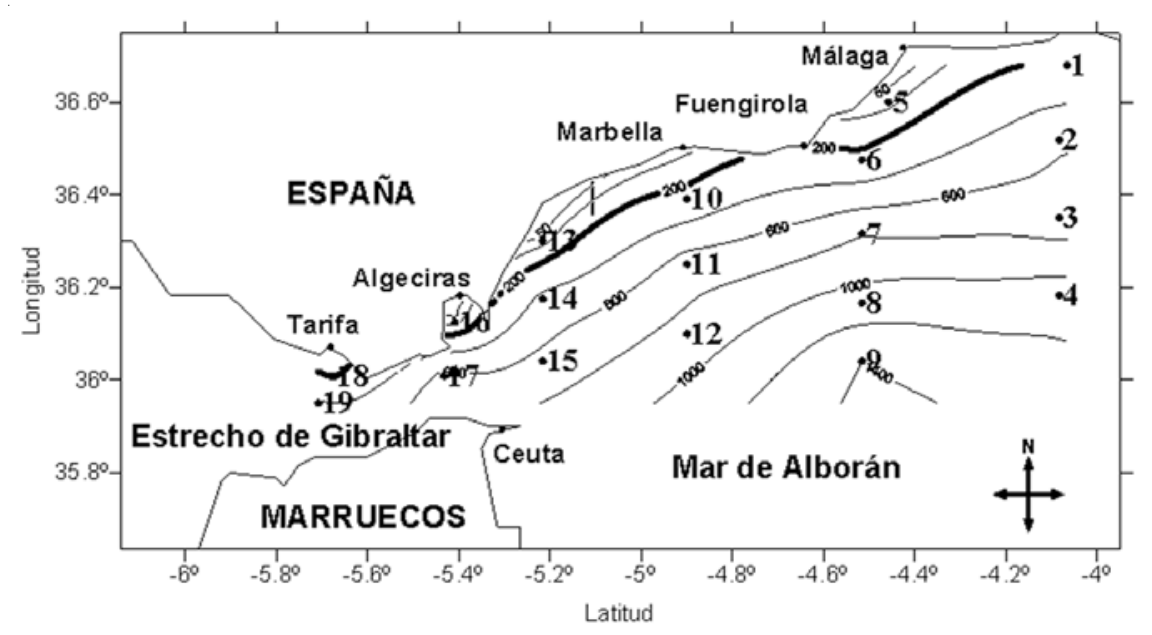

Figura 1

Estaciones muestreadas y topografía en el mar de Alborán y estrecho de Gibraltar

Sampling stations and bathymetry in the Alboran Sea and Gibraltar Strait

1994, Gómez et al. 2000) y notorias diferencias en las comunidades zooplanctónicas en la zona de divergencia (Rodríguez et al. 1982). Por otro lado, entre la plataforma y el mar abierto se detectaron intercambios de ictioplancton en ambas direcciones, fenómeno que se consideró favorecido por la estrechez de la plataforma continental en el mar de Alborán (Rubín et al. 1997a).

En el presente trabajo se pretende avanzar en la comprensión del citado proceso, estudiando principalmente la influencia de las masas de agua sobre la composición y distribución de las larvas de peces en el área comprendida entre el Estrecho y sector noroeste del mar de Alborán, delimitados aproximadamente por las localidades de Tarifa y Torre del Mar.

\section{Material y métodos}

El material de estudio procede de las campañas estivales 'Ictio.Alborán', del Instituto Español de Oceanografía, realizadas con el B.O. Francisco de Paula Navarro en los meses de julio de 1993 y de 1994. Todos los muestreos fueron diurnos. Se empleó una batisonda-CTD SeaBird $\circledast$ SBE-25, para la caracterización hidrográfica de las estaciones de muestreo, desde la superficie hasta los $500 \mathrm{~m}$ de profundidad. Los arrastres planctónicos fueron del tipo doble-oblicuo, desde la superficie hasta los 100 $\mathrm{m}$, con una red Bongo (mallas 250 y $335 \mu \mathrm{m}$ ) dotada de dos flujómetros General Oceanics $₫ 2030 \mathrm{R}$ y un profundímetro Mega Sport $\AA_{140 L M}$. Las muestras se conservaron en formol al 4\%, previamente tamponado con bórax. Las capturas de la malla de $335 \mu \mathrm{m}$ se emplearon para la identificación taxonómica del ictioplancton y los resultados se estandarizaron con el método de Smith \& Richardson (1979). Considerando los resultados preliminares publicados por separado para ambas campañas (Rubín et al. 1997a, b) se seleccionaron 19 estaciones comunes entre los dos muestreos para el presente trabajo (Fig. 1), sobre las que se realizó una diferente exploración de los datos hidrológicos e ictioplanctónicos, subdividiendo los taxones larvarios en 'Neríticos' (epipelágicos y bentónicos) y 'Oceánicos' (mesopelágicos). Larvas recién eclosionadas (estadio de preflexión) que no fueron identificadas se han denominadas taxa indeterminado.

Para cada campaña se llevaron a cabo dos tipos de dendrogramas, analizando por separado las agrupaciones de las 19 estaciones y los mismos 13 taxones seleccionados. Esos taxones se eligieron teniendo en cuenta un porcentaje numérico superior a 3\% (11 taxones) en cualquier de los dos años, y aquellos otros que fueron dominantes en alguna estación concreta de cada año (Blenniidae y Engraulis encrasicolus). Se empleó el coeficiente de distancia de Manhatan como medida de la disimilaridad, y el método de Ward como algoritmo de agregación (Pielou 1984). Las matrices de la comunidad se constituyeron de la siguiente forma: en columnas las unidades de muestreo, en filas los taxones larvarios dominantes, y como entradas las abundancias relativas $\left(\mathrm{n}^{\mathrm{o}}\right.$ individuos $10 \mathrm{~m}^{-2}$ ). Para graficar las masas de agua se siguió la nomenclatura propuesta por Cano et al. (1997) y Rubin et al. (1997a,b) para el área: st (propia del Estrecho, presencia de Agua Central Noratlántica bajo la capa superficial, con salinidad de 35,1-36,7 y temperatura de $8-19^{\circ} \mathrm{C}$ ), a (Agua Superficial Atlántica, con salinidad de 36,4-36,5 y temperatura de $15-20^{\circ} \mathrm{C}$ ), m (Agua 
Superficial Mediterránea, salinidad superior a 37,2 ups y temperatura superior a $22^{\circ} \mathrm{C}$ ), ma (con salinidad de 36,6-37 ups) y am (intermedia entre ma y a).

\section{Resultados}

\section{Masas de agua}

En 1993, las isotermas tuvieron un rango de variación entre los 18 y $22^{\circ} \mathrm{C}$ la salinidad varió entre 36,5 y 37 ups, en función de una mayor presencia de aguas de mezcla. Sin embargo, en 1994, la mayor presencia del flujo atlántico entrante determinó la presencia de aguas menos cálidas (entre 17 y $20^{\circ} \mathrm{C}$ ) y menos salinas (entre 36,3 y 36,9 ups). En ambos años se produjeron grandes cambios hidrológicos, tanto a nivel costero como oceánico (Fig. 2). Sin embargo, la situación que podemos considerar predominante para la zona de estudio es la observada en el año 1993: las masas de agua st limitadas al Estrecho, y en el mar de Alborán dominio de las aguas de carácter más mediterráneo, con mayor temperatura y salinidad en el sector costero y de las de naturaleza más atlántica, con menor temperatura $\left(<20^{\circ} \mathrm{C}\right) \mathrm{y}$ salinidad (<36,5 ups). En 1994 se detectó en mar abierto una ampliación espacial (horizontal y vertical) hacia el E de las masas de agua st y hacia el E y NE de la del tipo a. El dominio del agua de origen atlántico fue máximo incluso
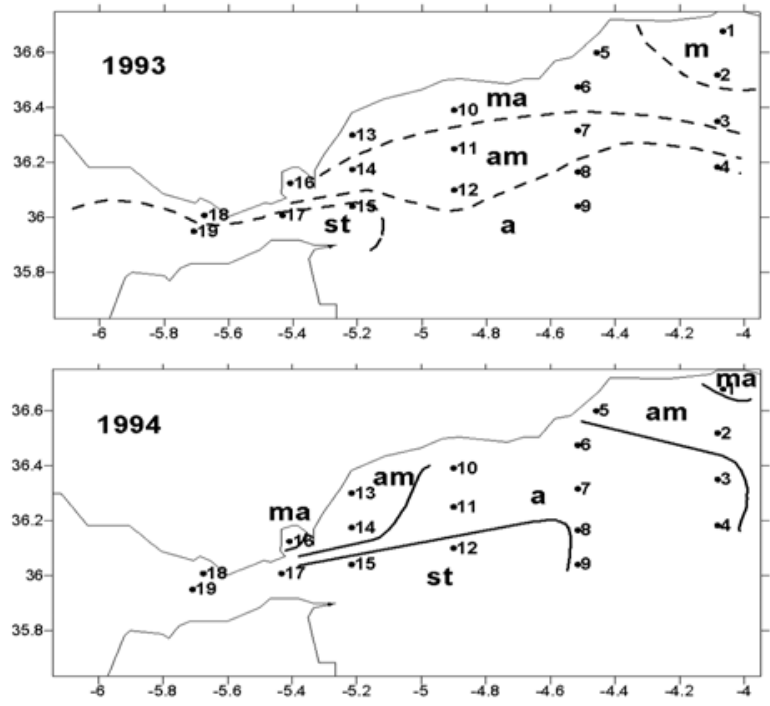

Figura 2

Tipos de masas de agua en el mar de Alborán y estrecho de Gibraltar: st (Agua del Estrecho), a (Agua Atlántica), m

(Agua Superficial Mediterránea); y aguas de mezcla: am (dominio atlántico) y ma (dominio mediterráneo)

Water masses in the Alboran Sea and Gibraltar Strait: st (Water of the Strait), a (Atlantic Water), m (Mediterranean Surface Water); and Mixed Waters: am (Atlantic Mediterranean Water) y ma (Mediterranean Atlantic Water)

Tabla 1

Masas de agua y larvas de peces dominantes en cada estación. Se señalan las estaciones más costeras (*) y los taxones neríticos. st (Agua del Estrecho), a (Agua Atlántica), m (Agua Superficial Mediterránea); y aguas de mezcla: am (dominio atlántico) y ma (dominio mediterráneo)

Water masses and dominant fish larvae in each station. (* coastal stations; neritic taxa). st (Strait Water), a (Atlantic Water), m (Mediterranean Surface Water); and mixed waters: am (Atlantic Mediterranean Water) y ma (Mediterranean Atlantic Water)

\begin{tabular}{|c|c|c|c|}
\hline Estación & $\begin{array}{c}\text { Masas de agua } \\
1993 / 1994\end{array}$ & $\begin{array}{l}\text { Larvas dominantes } \\
1993\end{array}$ & $\begin{array}{l}\text { Larvas dominantes } \\
1994\end{array}$ \\
\hline $1 *$ & $\mathrm{~m} / \mathrm{ma}$ & Sparidae & Cepola rubescens \\
\hline 2 & $\mathrm{~m} / \mathrm{am}$ & Benthosema glaciale & Benthosema glaciale \\
\hline 3 & $\mathrm{am} / \mathrm{a}$ & Ceratoscopelus maderensis & Maurolicus muelleri \\
\hline 4 & $\mathrm{a} / \mathrm{a}$ & Capros aper & Hygophum benoiti \\
\hline $5^{*}$ & $\mathrm{ma} / \mathrm{am}$ & $\underline{\text { Blenniidae }}$ & Bothidae \\
\hline 6 & $\mathrm{ma} / \mathrm{a}$ & Benthosema glaciale & Maurolicus muelleri \\
\hline 7 & $\mathrm{am} / \mathrm{a}$ & Ceratoscopelus maderensis & Maurolicus muelleri \\
\hline 8 & $\mathrm{a} / \mathrm{a}$ & Capros aper & Capros aper \\
\hline 9 & $\mathrm{a} / \mathrm{a}$ & Hygophum benoiti & Hygophum benoiti \\
\hline $10 *$ & $\mathrm{ma} / \mathrm{a}$ & Cepola rubescens & Ceratoscopelus maderensis \\
\hline 11 & $\mathrm{am} / \mathrm{a}$ & Myctophum punctatum & Maurolicus muelleri \\
\hline 12 & $\mathrm{am} / \mathrm{st}$ & Ceratoscopelus maderensis & Engraulis encrasicolus \\
\hline $13^{*}$ & $\mathrm{ma} / \mathrm{am}$ & Callyonimidae & Gobiidae \\
\hline 14 & $\mathrm{am} / \mathrm{am}$ & Maurolicus muelleri & Maurolicus muelleri \\
\hline 15 & st / st & Maurolicus muelleri & Maurolicus muelleri \\
\hline $16^{*}$ & $\mathrm{ma} / \mathrm{ma}$ & Callionymidae & $\underline{\text { Gobiidae }}$ \\
\hline 17 & st / st & Capros aper & $\underline{\text { Sparidae }}$ \\
\hline $18^{*}$ & st / a & Capros aper & Engraulis encrasicolus \\
\hline 19 & st / st & Capros aper & Engraulis encrasicolus \\
\hline
\end{tabular}


en la zona costera, donde las referidas aguas más atlánticas (a) invadieron el sector nerítico central (Marbella-Fuengirola) “el más saliente y cercano a las aguas oceánicas" desplazando hacia los sectores distales las aguas de mezcla (am y ma) y reduciendo la presencia de las más mediterráneas (ma) a dos localizaciones extremas muy distantes entre sí (bahía de Algeciras y Torre del Mar). El tipo de agua presente en cada estación se detalla en la Tabla 1, donde se puede comprobar que el cambio interanual fue mayoritario y afectó a 11 estaciones ( $58 \%$ del total).

\section{Composición faunística y abundancia de larvas de peces}

Se identificó un total de 5831 larvas (3545 en 1993 y 2286 en 1994) que fueron clasificadas en 34 categorías sistemáticas, pertenecientes a 25 familias (Tabla 2). Los individuos se encontraron en estadio de preflexión en un

Tabla 2

Lista taxonómica de larvas de peces identificadas, su origen ( $\mathrm{N}$ - nerítica; OC - oceánica), abundancias absoluta y relativa (\%). (Sp.: familia con un morfotipo, Spp.: familia con más de un morfotipo)

List of larval fish taxa identified their origin ( $\mathrm{N}$ - neritic; OC - oceanic), absolute and relative abundances (\%).

(Sp.: family with one morphotype, Spp.: family with more than one morphotype)

\begin{tabular}{|c|c|c|c|c|}
\hline Familia & & Origen & $1993(\%)$ & $1994(\%)$ \\
\hline CLUPEIDAE & Sardinella aurita & $\mathrm{N}$ & $1(0,03)$ & $2(0,08)$ \\
\hline ENGRAULIDAE & Engraulis encrassicolus & $\mathrm{N}$ & $22(0,53)$ & $69(2,78)$ \\
\hline GONOSTOMATIDAE & Cyclothone braueri & $\mathrm{OC}$ & $43(1,43)$ & $6(0,23)$ \\
\hline STERNOPTYCHIDAE & Maurolicus muelleri & $\mathrm{OC}$ & $132(3,86)$ & $181(6,57)$ \\
\hline PHOTICHTHYIDAE & Vinciguerria sp. & $\mathrm{OC}$ & $10(0,32)$ & $17(0,80)$ \\
\hline STOMIIDAE & Stomias boa boa & $\mathrm{OC}$ & $16(0,53)$ & $19(0,80)$ \\
\hline PARALEPIDIDAE & Lestidiops sp. & $\mathrm{OC}$ & $22(0,68)$ & $19(0,78)$ \\
\hline \multirow[t]{8}{*}{ MYCTOPHIDAE } & Benthosema glaciale & $\mathrm{OC}$ & $274(7,54)$ & $150(6,72)$ \\
\hline & Ceratoscopelus maderensis & $\mathrm{OC}$ & $256(7,19)$ & $111(4,53)$ \\
\hline & Diaphus holti & $\mathrm{OC}$ & $97(2,68)$ & $22(1,03)$ \\
\hline & Hygophum benoiti & $\mathrm{OC}$ & $498(14,20)$ & $126(6,26)$ \\
\hline & Hygophum hygomii & $\mathrm{OC}$ & $90(2,43)$ & $2(0,11)$ \\
\hline & Lampanyctus pusilos & $\mathrm{OC}$ & $46(1,25)$ & $26(1,29)$ \\
\hline & Lobianchia dofleini & $\mathrm{OC}$ & $1(0,03)$ & $2(0,11)$ \\
\hline & Myctophum punctatum & $\mathrm{OC}$ & $138(3,86)$ & $41(2,07)$ \\
\hline OPHIDIIDAE & Sp. & $\mathrm{N}$ & $0(0,00)$ & $1(0,04)$ \\
\hline GADIDAE & Sp. & $\mathrm{OC}$ & $1(0,03)$ & $0(0,00)$ \\
\hline MACROURIDAE & Sp. & $\mathrm{OC}$ & $1(0,03)$ & $0(0,00)$ \\
\hline CAPROIDAE & Capros aper & $\mathrm{OC}$ & $316(10,84)$ & $71(3,52)$ \\
\hline MACRORAMPHOSIDAE & Macroramphosus scolopax & $\mathrm{OC}$ & $0(0,00)$ & $1(0,04)$ \\
\hline SCORPAENIDAE & Sp. & $\mathrm{N}$ & $1(0,03)$ & $1(0,04)$ \\
\hline \multirow[t]{4}{*}{ SERRANIDAE } & & $\mathrm{N}$ & $10(0,32)$ & $33(1,44)$ \\
\hline & Serranus cabrilla & $\mathrm{N}$ & & \\
\hline & Serranus hepatus & $\mathrm{N}$ & & \\
\hline & Anthias sp. & $\mathrm{N}$ & & \\
\hline CARANGIDAE & Trachurus sp. & $\mathrm{N}$ & $42(1,38)$ & $41(2,02)$ \\
\hline \multirow[t]{2}{*}{ SPARIDAE } & & $\mathrm{N}$ & $114(3,16)$ & $56(2,42)$ \\
\hline & Sparus pagrus & $\mathrm{N}$ & & \\
\hline CEPOLIDAE & Cepola rubescens & $\mathrm{N}$ & $101(2,60)$ & $68(3,95)$ \\
\hline \multirow[t]{2}{*}{ LABRIDAE } & & $\mathrm{N}$ & $16(0,43)$ & $74(2,96)$ \\
\hline & Coris julis & $\mathrm{N}$ & & \\
\hline BLENNIIDAE & Spp. & $\mathrm{N}$ & $44(0,92)$ & $19(1,05)$ \\
\hline CALLIONYMIDAE & Callionymus spp. & $\mathrm{N}$ & $95(2,30)$ & $57(3,00)$ \\
\hline GOBIIDAE & Spp. & $\mathrm{N}$ & $60(1,54)$ & $63(3,03)$ \\
\hline SCOMBRIDAE & Sarda sarda & $\mathrm{N}$ & $0(0,00)$ & $1(0,04)$ \\
\hline BOTHIDAE & Arnoglossus spp. & $\mathrm{N}$ & $86(2,61)$ & $66(3,33)$ \\
\hline ACHIRIIDAE & Sp. & $\mathrm{N}$ & $18(0,43)$ & $6(0,28)$ \\
\hline Taxa indeterminado & & $\mathrm{N}$ & $408(10,20)$ & $206(9,78)$ \\
\hline Taxa indeterminado & & $\mathrm{OC}$ & $586(16,58)$ & $729(28,86)$ \\
\hline TOTAL & & & $3545(100)$ & $2286(100)$ \\
\hline
\end{tabular}


alto porcentaje, lo que incrementó el porcentaje de taxa nerítico y oceánico indeterminados $($ media $=32,7 \%)$.

En ambos años se mantuvo un predominio numérico de las larvas de especies oceánicas, (mesopelágicas), con $73,5 \%$ de la captura total en 1993 y 63,7\% en 1994 . Considerando el conjunto de taxa más abundantes (Tabla 2), en 1993 cinco grupos oceánicos representaron el 43,6\% del total larvario (Hygophum benoiti, Capros aper, Benthosema glaciale, Ceratoscopelus maderensis y Maurolicus muelleri). En 1994 esos mismos taxones estuvieron entre los seis más abundantes, aunque disminuyó considerablemente su presencia (al 27,6\%) y cinco especies neríticas (costeras o de plataforma) aumentaron: Cepola rubescens, Bothidae, Gobiidae, Callionymidae y Labridae (al 16,27\%). También en ambos años las 19 estaciones resultaron positivas para la presencia larvaria, aunque el rango de abundancia fue más amplio en 1993 (59-6509 larvas $10 \mathrm{~m}^{-2}$, media = 1581 larvas $\left.10 \mathrm{~m}^{-2}\right)$ que en $1994\left(80-3173\right.$ larvas $10 \mathrm{~m}^{-2}$, media $=1042$ larvas $10 \mathrm{~m}^{-2}$ ).

\section{Distribución espacial de taxones}

En ambos años, el número total de taxones fue muy similar (29-30 taxones) y en la distribución horizontal (Fig. 3) se detectaron valores mínimos en el sector occidental $(\leq 10)$ y máximos en el oriental $(>16)$ del mar de Alborán. Sin embargo, únicamente en 1994 entre ambas agregaciones orientales se creó un área de valores bajos (<12). La amplia distribución espacial de los taxa costeros en 1993 (en las cinco estaciones más neríticas de Alborán y sector oceánico al oriente) se redujo considerablemente en 1994, desapareciendo el agrupamiento de mar abierto y extendiéndose las larvas oceánicas (Fig. 3) al sector nerítico de Marbella (est. 10). Por ello, en esa estación, la naturaleza de las cuatro especies más abundantes cambió totalmente: de costeras en 1993 (Cepola rubescens, Callionymidae, Gobiidae y Sparidae) a oceánicas en 1994 (Ceratoscopelus maderensis, Benthosema glaciale, Maurolicus muelleri y Lampanyctus sp.).

En el taxon costero Engraulis encrasicolus (boquerón), se detectó grandes cambios interanuales: en 1994 tuve una densidad media del 8,4 ind $10 \mathrm{~m}^{-2}(2,78 \%)$ $\mathrm{y}$ en $1993 \mathrm{del} 29,1$ ind $10 \mathrm{~m}^{-2}(0,53 \%)$. Concretamente, se detectaron cambios interanuales en las zonas de mayor agregación de larvas de E. encrasicolus, desde aguas más mediterráneas en verano de 1993 (al norte -bahía de Málaga- y al sureste), cambiando a aguas más atlánticas (al sur y suroeste) durante verano de 1994 (Fig. 4).
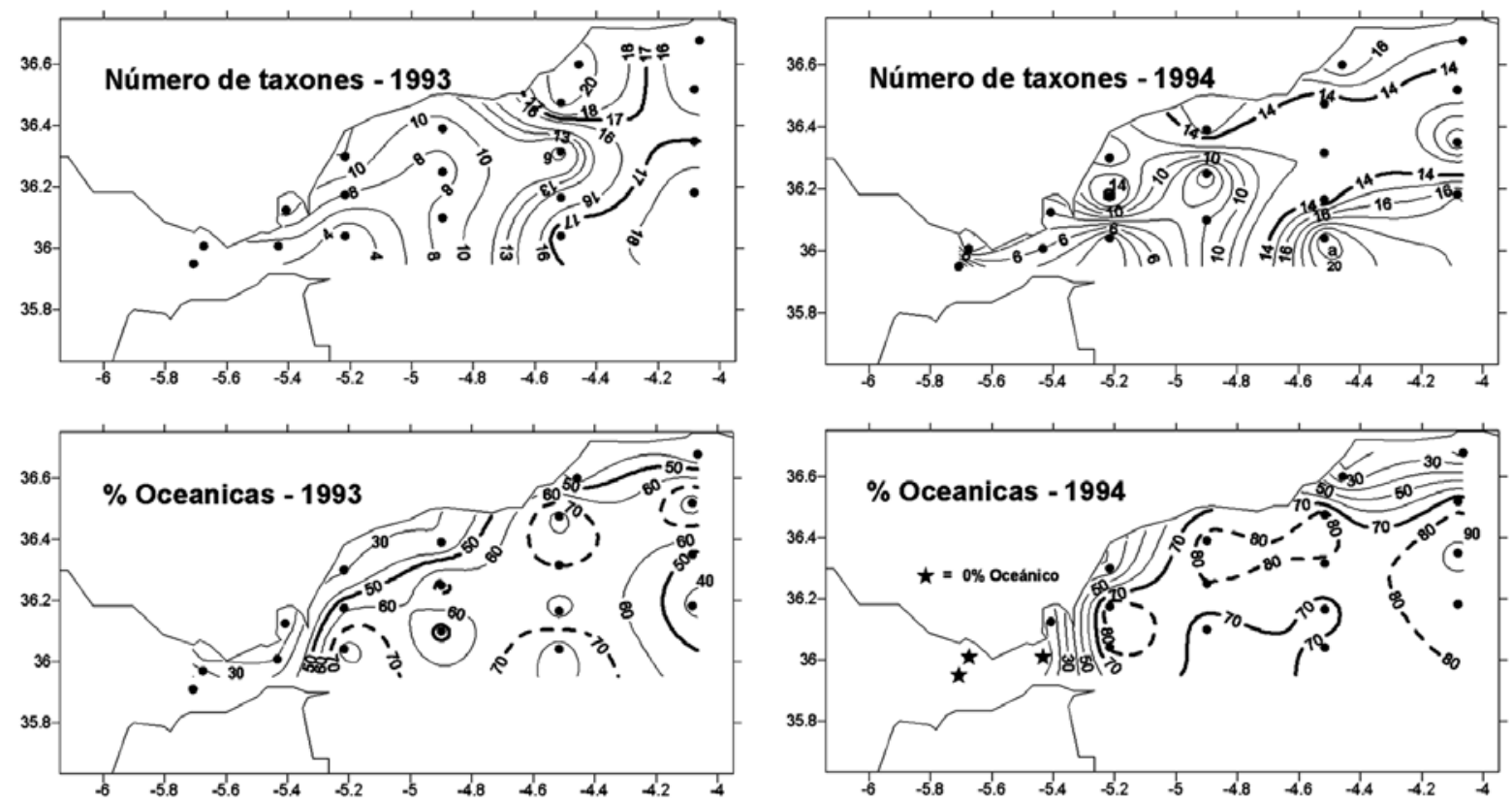

Figura 3

Distribución espacial del número total de taxones larvarios y porcentaje de taxones oceánicos en el mar de Alborán y estrecho de Gibraltar

Spatial distribution of total number of taxa and relative abundance of oceanic taxa in the Alboran Sea and Gibraltar Strait 

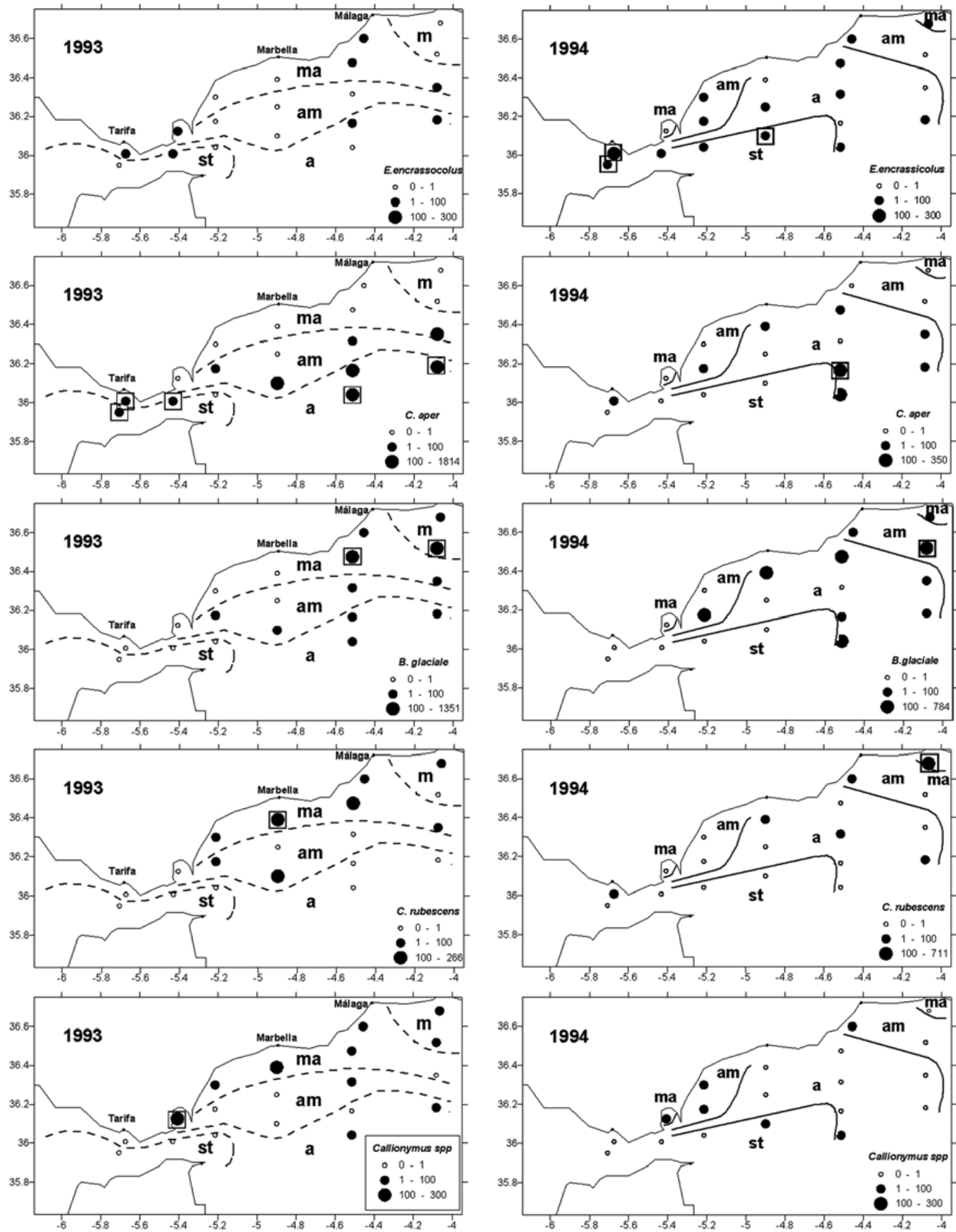

Figura 4

Distribución espacial (ind $10 \mathrm{~m}^{-2}$ ) de algunos taxa indicadores de masa de agua (E. encrassicolus, C. aper, B. glaciale, C. rubescens, Callyonimus spp.) en el mar de Alborán y estrecho de Gibraltar. Se señalan las estaciones donde el taxón ha sido dominante (\%)

Spatial distribution (ind $10 \mathrm{~m}^{-2}$ ) of some water mass taxa indicators (E. encrassicolus, C. aper, B. glaciale, C. rubescens, Callyonimus spp.) in the Alboran Sea and Gibraltar Strait. Stations where the taxon has been dominant are indicated (\%) 


\section{Agrupaciones de estaciones y de taxones}

Los mismos 13 taxones seleccionados para los dendrogramas de ambos años, representaron una abundancia global mas elevada en 1993 (61,2\% del total larvario) que en 1994 (49,2\%).

En 1993 (Fig. 5), las agrupaciones reflejaron una subdivisión latitudinal del área de estudio en los sectores litoral (norte) y oceánico (sur). Las cinco estaciones más costeras de Alborán quedaron agrupadas en el sector litoral, donde dominaron los taxones neríticos y éstos estuvieron asociados a las masas de agua más mediterráneas: $\mathrm{m}$ (Bothidae y Sparidae) y ma ( $C$. rubescens, Gobiidae, Callyonimidae, Blenniidae y $E$. encrasicolus). Por otra parte, en el sector de mar abierto, con dominancia de los taxones oceánicos $\mathrm{y}$, salvo el caso de Benthosema glaciale (ma), los restantes se relacionaron con las aguas más atlánticas: st (C. aper), a
(H. benoiti) y am (C. maderensis, $M$. punctatum y $M$. muelleri).

Durante el verano de 1994 se encontró una situación muy diferente, con una mezcla entre los costeros y oceánicos, reflejada en los dendrogramas (Fig. 5). Éstos marcaron una subdivisión longitudinal del área de estudio entre los sectores este y oeste, perpendiculares a la línea de costa. Por ese motivo, las cinco estaciones más costeras de Alborán dejaron de estar agrupadas, pues las dos más occidentales se agregaron con la costera del Estrecho (est. 18). Las masas de agua de ese sector occidental se asociaron principalmente a taxones costeros (cinco taxa), con un único taxón oceánico Myctophum punctatum (am). Situación contraria en el sector oriental, donde las masas de agua portaron mayoritariamente taxones oceánicos (cinco taxa), con solo dos taxones costeros: Callyonimidae y C. rubescens (ma).
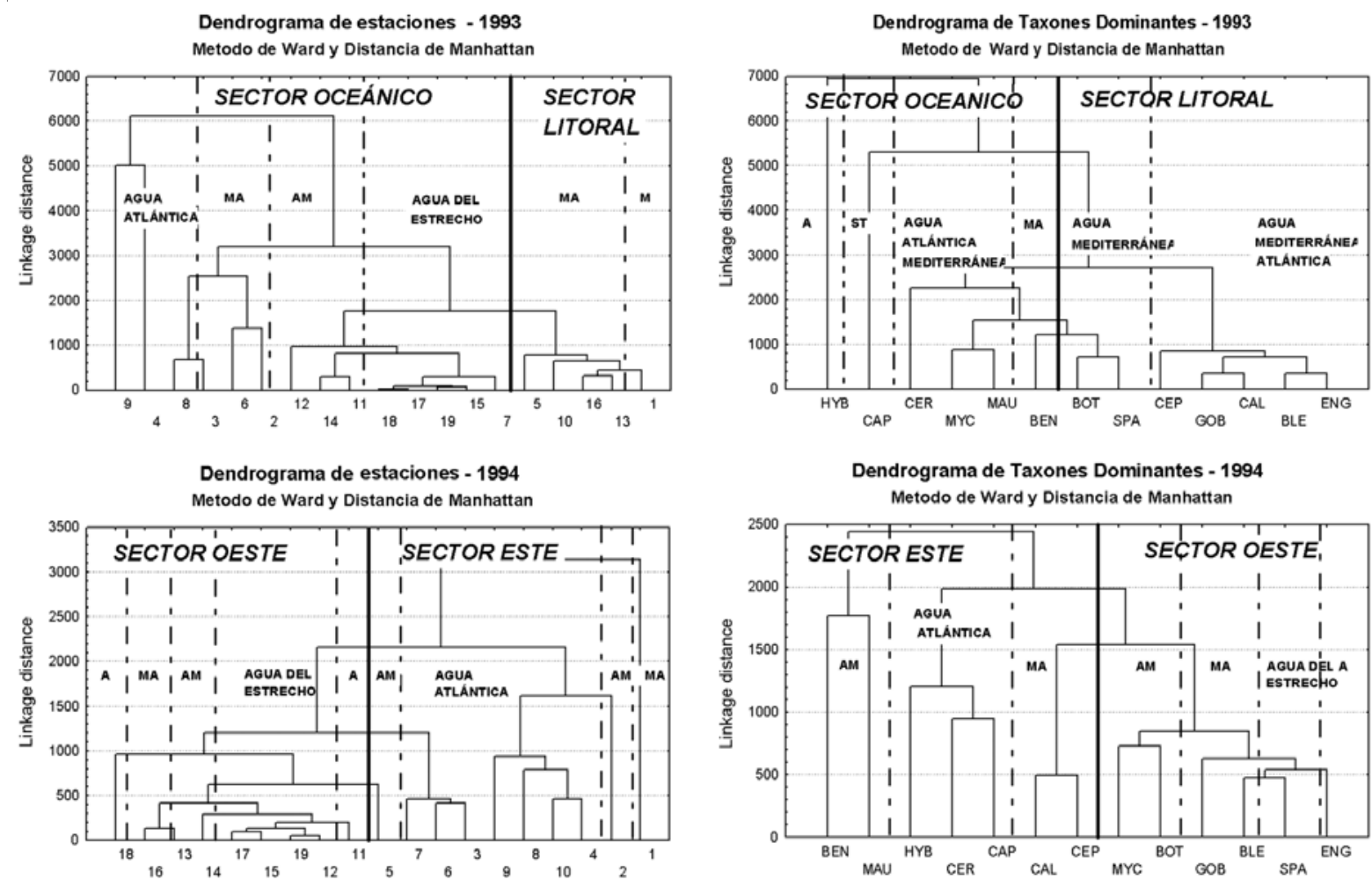

Figura 5

Agrupamientos de estaciones y de taxones, con indicación de las masas de agua asociadas (Ben = Benthosema glaciale, Ble $=$ Blenniidae, Bot $=$ Bothidae, Cal $=$ Callyonimidae, Cap= Capros aper, Cep $=$ Cepola rubescens, Cer $=$ Ceratoscopelus maderensis; Eng = Engraulis encrassicolus, Gob = Gobiidae, Hyb = Hygophum benoiti, Mau = Maurolicus muelleri, Myc $=$ Myctophum punctatum, y Spa $=$ Sparidae)

Groups of stations and taxa with associated water masses $(\mathrm{Ben}=$ Benthosema glaciale, $\mathrm{Ble}=$ Blenniidae, Bot $=$ Bothidae, $\mathrm{Cal}=$ Callyonimidae, $\mathrm{Cap}=$ Capros aper, $\mathrm{Cep}=$ Cepola rubescens, $\mathrm{Cer}=$ Ceratoscopelus maderensis; Eng = Engraulis encrassicolus, $\mathrm{Gob}=$ Gobiidae, Hyb = Hygophum benoiti, Mau = Maurolicus muelleri, Myc = Myctophum punctatum, y Spa = Sparidae) 


\section{Discusión}

En el Mediterráneo occidental se han realizado estudios para conocer la composición y abundancia del ictioplancton, en aguas neríticas y oceánicas, tanto en mar de Alborán (Rodríguez 1990, Rubín et al. 1997a y b; Rubín et al. 1994), como en el mar Menor (Bordehore et al. 2001, Vidal-Peñas et al. 2001) y en la costa catalana (Sabatés 1990). Sin embargo, del mar de Alborán, la información que se disponía, en lo referente a comunidades de ictioplancton, no relacionaba las diferencias en la composición de especies con la naturaleza de las masas de agua.

Los cambios hidrológicos interanuales que influyeron en la asociación entre larvas de peces y masas de agua fueron los valores superficiales mas bajos de temperatura y de salinidad, verificados en 1994. La variabilidad estacional e interanual de las masas de agua en Alborán es un hecho comprobado a nivel de cuenca (Cano 1978, Gil 1990). En este trabajo se confirma, a nivel de estación de muestreo, esa gran variabilidad hidrológica interanual en la zona de estudio para las dominantes masas de agua y taxones. Sin embargo, a nivel general se mantuvo en el área un número de taxones muy similar (29-30) y un predominio equivalente de los taxones oceánicos. Ese predominio de taxones oceánicos probablemente se vea favorecido por la estrechez de la plataforma continental, circunstancia que también favorecería el intercambio larvario entre las comunidades neríticas y oceánicas (Rubín et al. 1997a). En otras regiones marinas las diferencias en la composición de especies también han sido atribuidas a la amplitud de plataforma (Doyle et al. 1994, Vidal-Peñas et al. 2001) y la naturaleza de las masas de agua (Chiu \& Hsyu 1994, Leis \& Miller 1976).

A causa de los cambios hidrológicos detectados, la amplia distribución espacial, a nivel nerítico, de los taxones costeros en 1993 se redujo considerablemente en 1994, las larvas oceánicas irrumpieron en el sector nerítico de Marbella. Por ello, en la estación 10, la naturaleza de las cuatro especies más abundantes cambió totalmente, y las costeras de 1993 (C. rubescens, Callionymidae, Gobiidae y Sparidae) se vieron sustituidas en 1994 por C. maderensis, B. glaciale y M. muelleri. Esas tres especies son habituales en la zona, aunque son de naturaleza muy diferente: ártico-boreal (B. glaciale), afloramiento (M. muelleri) y templado-subtropical ( $C$. maderensis). El hecho de que esta última fuera dominante en 1993 en el agua atlántica (a), al sur del paralelo $36^{\circ} \mathrm{N}$ (Rubín et al. 1997a), permite relacionarla en 1994 con la irrupción de esa masa de agua en el sector nerítico. En el sudeste ibérico, la elevada abundancia de Myctophidae, cerca de la costa, lleva a sugerir una intrusión de agua oceánica (Vidal-Peñas et al. 2001). También Rodríguez (1990) detectó que en épocas de menor estratificación térmica y épocas de afloramientos había mayores abundancias de mesopelágicos cerca de la costa, en el mar de Alborán.

En el golfo de Cádiz, en verano, han sido identificadas una asociación costera (Gobiidae, Labridae, Engraulis encrasicolus, Sparidae y Callionymidae) y otra de plataforma (Sardinela aurita, Trachurus spp.) donde han predominado los taxones neríticos (Mafalda \& Rubin 2006). En la reserva marina de Tabarca, sudeste ibérico, también la comunidad estuvo muy influida (99\%) por la presencia de taxones neríticos, donde en el verano se destacan Gobiidae, Gobiesocidae y Clupeidae (Bordehore et al. 2001). Mientras que en las Islas Hormigas (sudeste ibérico), ha sido verificada una composición ictioplanctónica semejante al mar de Alborán, donde las larvas de peces que excavan sobre la plataforma (Sardina pilchardus, Gobiidae y Sparidae) dominaron las estaciones someras, mientras que las larvas de Myctophidae (Benthosema glaciale y Lampanyctinae) dominaron las estaciones situadas en el talud continental. Los resultados del sudeste ibérico sugieren la presencia de un frente halino, que limita la dispersión de las larvas según un eje costa - mar abierto (Vidal-Peñas et al. 2001), similar al descrito en los mares Catalán (Sabatés 1990) y Balear (La Violette et al. 1990).

En el mar de Alborán, a diferencia de otras áreas costeras de la península ibérica (Drake \& Arias 1991, Bordehore et al. 2001, Mafalda \& Rubin 2006), la poca densidad de larvas de especies costeras, a favor de las oceánicas, muestra una baja influencia de la ictiofauna local, debido a una elevada influencia de aguas profundas o lejanas a la costa.

Las consecuencias de una mayor actividad del chorro Atlántico en verano de 1994 se reflejaron en la presencia de larvas de peces mesopelágicos sobre la plataforma continental frente a Fuengirola. Probablemente las masas de aguas transportaron larvas oceánicas al borde costero, y las larvas neríticas que se encontraban en aguas sobre la plataforma fueron advectadas hacia los bordes de la cuenca. Estos resultados sugieren que la estructura de las asociaciones larvarias, es regulada por los cambios en la hidrodinámica local que ponen en manifiesto la importancia de la deriva de masas de agua de mar abierto hacia la costa.

\section{Agradecimientos}

Los autores expresan gratitud a todos los participantes de las campañas 'Ictio.Alborán' y a la CAPES (Ministério da Educaçao, Brasil) por la concesión de una beca de 
estancia postdoctoral (Ref.: BEX: 0762-03-2) en el Centro Oceanográfico de Málaga-Fuengirola, Instituto Español de Oceanografía (IEO). Los autores agradecemos los comentarios y sugerencias de los revisores para mejorar este manuscrito.

\section{Literatura citada}

Ballester A \& J Zavatti. 1983. Aspectos químicos del afloramiento de Alborán. Resultados del Proyecto 793020 del Comité Hispano-Norteamericano CHN: 334-355.

Bordehore C, T Bayle Sempre \& AA Ramos Esplá. 2001. Composición y variabilidad del ictioplancton costero en la reserva marina de Tabarca, sudeste ibérico. Boletín del Instituto Español de Oceanografía 17(1-2): 61-71.

Cano N. 1978. Hidrología del mar de Alborán en primaveraverano. Boletín del Instituto Español de Oceanografía 248(4): 51-66.

Cano N \& J García Lafuente. 1991. Corrientes en el litoral malagueño. Baja frecuencia. Boletín del Instituto Español de Oceanografía 7(2): 59-77.

Cano N, J García Lafuente, A Hernández-Guerra, JMA Blanco \& J Escánez. 1997. Hidrología del mar de Alborán en julio de 1993. Publicaciones Especiales del Instituto Español de Oceanografía 24: 9-26.

Cortés MD, J Gil \& A García. 1985. General distribution of chlorophyll, temperature and salinity in the NW sector of the Alboran Sea (August, 1982). Rapport Commission Institut Mer Méditerranéenne 29(9): 135-136.

Cheney RE \& RA Doblar. 1982. Structure and variability of the Alboran Sea frontal system. Journal of Geophysical Research 87: 585-594.

Chiu TS \& YH Hsyu. 1994. Interannual variation of ichthyoplankton density and species composition in the waters off northeastern Taiwan. Marine Biology 119: 441448.

Doyle MJ, WW Morse \& AW Kendall. 1994. A comparison of larval fish assemblages in the temperate zone of the northeast Pacific and northwest Atlantic Oceans. Bulletin of Marine Science 53: 630-644.

Drake P \& AM Arias. 1991. Ichthyoplankton of a shallowcoastal inlet in south-west Spain: factors contributing to colonization and retention. Estuarine Coastal and Shelf Science 32: 347-364.

García Lafuente J, N Cano, M Vargas, JP Rubín \& A Hernández-Guerra. 1998. Evolution of the Alborán Sea hydrographic structures during July 1993. Deep-Sea Research I 45: 39-65.

García Lafuente J \& N Cano. 1994. Tidal dynamics and associated features of the nortwestern shelf of the Alboran Sea. Continental Shelf Research 14(1): 1-21.

Gil J. 1985. Hidrología de la costa suroccidental entre Motril y Estepona, en marzo de 1982. Informes Técnicos del Instituto Español de Oceanografía 33: 1-7.

Gil J. 1990. Hidrografía de la plataforma continental española mediterránea y golfo de León, en mayo-junio de 1988. Informes Técnicos del Instituto Español de Oceanografía 81: 1-124.

Gómez F, N González, F Echevarría \& CM García. 2000. Distribution and fluxes of dissolved nutrients in the Strait of Gibraltar and its relationships to microphytoplankton biomass. Estuarine, Coastal and Shelf Science 51(4): 439449.

La Violette PE, J Tintoré \& J Font. 1990. The surface circulation of the Balearic Sea. Journal of Geophysical Research 95(C2): 1559-1568.

Leis JM \& JM Miller. 1976. Offshore distributional patterns of Hawaiian fish larvae. Marine Biology 36: 359-367.

Mafalda Jr. P \& JP Rubín. 2006. Interannual variation of larval fish assemblages in the Gulf of Cadiz (SW Iberian Peninsula) in relation to summer oceanographic conditions. Brazilian Archives of Biology and Technology 49(2): 287296.

Parada M \& M Cantón. 1998. The spatial and temporal evolution of thermal structures in the Sea Mediterranean basin. International Journal of Remote Sensing 19 (11): 2119-2131.

Pielou EC. 1984. The Interpretation of Ecological Data: a Primer on Classification and Ordination, 263 pp. Wiley, New York.

Rodríguez J. 1990. Contribución al conocimiento del ictioplancton del mar de Alborán. Boletín del Instituto Español de Oceanografía 6: 1-20.

Rodríguez V, B Bautista, JM Blanco, FL Figueroa, N Cano \& J Ruiz. 1994. Hydrological structure, optical characteristics and size distribution of pigments and particles at a frontal station in the Alboran Sea. Scientia Marina 58: 31-41.

Rodríguez J, A García \& V Rodríguez. 1982. Zooplanktonic communities of the divergence zone in the Northwestern Alboran Sea. Marine Ecology 3(2): 133-142.

Rubín JP, J Gil, V Rodríguez, JM Blanco, F Echevarría J Rodríguez, F Jiménez-Gómez \& B Bautista. 1994. Relaciones entre distribución ictioplanctónica hidrología, biomassa y pigmentos fitoplanctónicos, oxígeno disuelto y nutrientes, en el mar de Alborán (julio 1992). Informes Técnicos del Instituto Español de Oceanografía 146: 7987. 
Rubín JP, N Cano, V Rodríguez, JMA Blanco, F JiménezGómez, J Rodríguez, J García Lafuente, F Echevarría, FJ Guerrero, J Escánez, A Hernández \& M Chbani. 1997a. Relaciones del ictioplancton con la hidrología, biomasa fitoplanctónica, oxígeno disuelto y nutrientes, en el mar de Alborán y estrecho de Gibraltar (julio 1993). Publicaciones Especiales del Instituto Español de Oceanografía 24: 75-84.

Rubín JP, N Cano, PArrate, J García, J Escánez, M Vargas \& F Hernández. 1997b. El ictioplancton, el mesozooplancton y el medio marino en el golfo de Cádiz, estrecho de Gibraltar y sector noroeste del mar de Alborán, en julio de 1994. Informes Técnicos del Instituto Español de Oceanografía 167: 1-48.

Sabatés A. 1990. Changes in the heterogeneity of mesoscale distribution patterns of larval fish associated with a shallow coastal hyaline front. Estuarine, Coastal and Shelf Science 30: 131-140.

Sarhan T, J García Lafuente, M Vargas, JM Vargas \& F Plaza. 2000. Upwelling mechanisms in the northwestern Alboran Sea. Journal of Marine Systems 23: 317-331.

Smith PE \& SL Richardson. 1979. Técnicas modelo para prospecciones de huevos y larvas de peces pelágicos. FAO. Documentos Técnicos de Pesca 175: 1-107.

Vidal Peñas I, F López Castejón \& J Mas Hernández. 2001. Influencia de la topografía submarina sobre la distribución horizontal de las comunidades de ictioplancton en el entorno de la reserva marina de cabo de Palos - islas Hormigas (sudeste ibérico). Boletín del Instituto Español de Oceanografía 17(1-2): 129-135.

Recibido el 3 de enero de 2007 y aceptado el 30 de noviembre de 2007 